\title{
Temperature-dependent Brillouin scattering studies of surface acoustic modes in $\mathrm{Nd}_{0.5} \mathrm{Sr}_{0.5} \mathrm{MnO}_{3}$
}

\author{
Md. Motin Seikha,b,*, Chandrabhas Narayana ${ }^{\mathrm{a}}$, Sachin Parashar $^{\mathrm{a}}$, A.K. Sood ${ }^{\mathrm{a}, \mathrm{c}}$ \\ ${ }^{a}$ Chemistry and physics of materials unit, Jawaharlal Nehru Center for Advanced Scientific Research, Jakkur P.O., Bangalore 560 064, India \\ ${ }^{\mathrm{b}}$ Solid Sate and Structural Chemistry Unit, Indian Institute of Science, Bangalore 560012 , India \\ ${ }^{\mathrm{c}}$ Department of Physics, Indian Institute of Science, Bangalore 560 012, India
}

\begin{abstract}
Brillouin scattering experiments are carried out to study the surface acoustic waves in $\mathrm{Nd}_{0.5} \mathrm{Sr}_{0.5} \mathrm{MnO}_{3}$ as a function of temperature in the range of 40-300 K covering the metal-insulator and charge-ordering phase transitions. The surface modes include surface Rayleigh wave, pseudo-surface acoustic wave (PSAW) and high velocity PSAW. The observed softening of the sound velocities for the surface modes below paramagnetic to ferromagnetic transition, $T_{\mathrm{c}}$ is related to the softening of the $\mathrm{C}_{44}$ elastic constant. The subsequent hardening of the sound velocity below the charge ordering transition temperature $T_{\text {co }}$ is attributed to the coupling of the acoustic phonon to the charge ordered state via long range ordering of the strong Jahn-Teller (JT) distortion.
\end{abstract}

\section{Introduction}

Doped manganites $\mathrm{A}_{1-x} \mathrm{~B}_{x} \mathrm{MnO}_{3}$ (where $\mathrm{A}$ and $\mathrm{B}$ are trivalent rare-earth and divalent alkaline earth ions, respectively) with the distorted pervoskite structure with three-dimensional network of $\mathrm{MnO}_{6}$ octahedra, have attracted considerable interest due to the discovery of negative colossal magnetoresistance (CMR) [1]. Apart from CMR, depending on the doping, applied pressure, A-site ionic radius, temperature and magnetic field the manganites show several fascinating properties such as coexistence of ferromagnetism and metallicity, orbital ordering, charge ordering and phase separation. Such physical properties can be attributed to the strong interplay among charge, spin, orbital and lattice degree of freedoms. The essence of

\footnotetext{
* Corresponding author. Address: Chemistry and physics of materials unit, Jawaharlal Nehru Center for Advanced Scientific Research, Jakkur P.O., Bangalore 500 064, India.

E-mail address: mseikh@jncasr.ac.in (M.M. Seikh).
}

paramagnetic insulator (PI) to ferromagnetic metal transition is captured within the framework of Zener doubleexchange (DE), where the ferromagnetic coupling between localized $\mathrm{Mn}_{2 \mathrm{~g}}$ spins is mediated by the hopping of $\mathrm{e}_{\mathrm{g}}$ electrons, which enables the avoidance of Hund's rule energy [2-4]. However, the DE model provides only a partial understanding of the manganites. It hardly explains quantitatively the large drop in resistivity below the ferromagnetic transition $T_{\mathrm{c}}$. This suggests that an understanding of these materials must include in addition to DE, strong electron correlations and electron-phonon interaction $[5,6]$. There is considerable experimental evidence to suggest the importance of the lattice via magnetic or lattice polaron mechanism in determining the electronic and magnetic properties of the manganites [7-9]. These experiments include neutron scattering [9] and isotopic shifts of $T_{\mathrm{c}}$ arising from the coupling of the charge carriers to Jahn-Teller polaron [10]. Despite many studies, a complete understanding of the unusual physical properties in pervoskite manganites is still lacking. 
The half-doped neodymium manganite, $\mathrm{Nd}_{0.5} \mathrm{Sr}_{0.5} \mathrm{MnO}_{3}$, on cooling undergoes a transition from the PI phase to the ferromagnetic metallic (FMM) phase around $260 \mathrm{~K}\left(T_{\mathrm{c}}\right)$ and on further cooling, shows a first order transition from the FMM phase to a charge ordered insulator (COI) phase at $\sim 150 \mathrm{~K}\left(T_{\mathrm{co}}\right)$. There is a structural transition from orthorhombic Pbnm to monoclinic $\mathrm{P} 2{ }_{1} / m$ at $T_{\mathrm{co}}$, whereas there is no structural transition associated with $T_{\mathrm{c}}$ [11]. This metal-insulator (MI) transition at $T_{\text {co }}$ has been assigned to real space ordering of the $\mathrm{Mn}^{3+}$ and $\mathrm{Mn}^{4+}$ ions, as in the case of Verwey transition in $\mathrm{Fe}_{3} \mathrm{O}_{4}$, where the mixed valent $\mathrm{Fe}^{2+}$ and $\mathrm{Fe}^{3+}$ ions ordering takes place [12] and was confirmed by neutron diffraction measurement [13]. The MI transition in $\mathrm{Nd}_{0.5} \mathrm{Sr}_{0.5} \mathrm{MnO}_{3}$ is accompanied by a spin and a $\mathrm{d}_{3 z^{2}-r^{2}}$ type orbital-ordering [11]. The charge-exchange (CE)-type anti-ferromagnetic structure is also realized in the COI phase. The charge-ordered anti-ferromagnetic insulating state collapses in an external magnetic field and the insulating anti-ferromagnetic phase transforms into a FMM phase $[14,15]$. These results point out that the stability of the COI state is related to the effective strength of the DE interaction. The stability of the COI state is sensitive to the commensurability of the charge carrier concentration [16]. This feature indicate that the stability of the COI state depends on the interactions and competions among the spin, charge and lattice degree of freedoms.

The occurrence of different types of electronic phases is well known in the rare earth manganites $[17,18]$. The coexistence of the three phases namely A-type, CE-type anti-ferromagnetic insulator and FM metallic phases has been reported both experimentally and theoretically in $\mathrm{Nd}_{0.5} \mathrm{Sr}_{0.5} \mathrm{MnO}_{3}[13,19,20]$. The existence of nanoscale regions of paramagnetic and ferromagnetic phases in $\mathrm{Nd}_{0.5} \mathrm{Sr}_{0.5} \mathrm{MnO}_{3}$ has been reported [21]. The appearance of the magnetic domain walls on cooling below $T_{\mathrm{c}}$ has been found in this system, which were oriented along the [100] and [110] directions and show a discontinuous jump on further cooling [22]. The appearance of the granular state of nanoclustures in the strongly correlated electron system is an intrinsic characteristic phenomenon, which would be associated with electronic phase separation $[17,18]$.

The relationship between the local lattice distortion, charge distribution and magnetism have been measured and discussed in the literature [23]. Sound velocity measurements at the transition point provide valuable information about the crystal lattice. In the ultrasound experiment, there is a significant renormalization of the sound velocity around $T_{\text {co }}$, which is related to the formation of the COI state in the presence of strong electron-lattice coupling with the static Jahn-Teller lattice distortion [24]. Unfortunately, however, there is no report of the sound velocity measurements at the $T_{\mathrm{c}}$ in $\mathrm{Nd}_{0.5} \mathrm{Sr}_{0.5} \mathrm{MnO}_{3}$. In this paper, we report surface sound velocity measurements in $\mathrm{Nd}_{0.5} \mathrm{Sr}_{0.5} \mathrm{MnO}_{3}$ in the temperature range of $40-300 \mathrm{~K}$ using Brillouin scattering. Brillouin scattering is a powerful non-destructive technique for the measurement of the surface and bulk acoustic modes. We observe substantial softening of the acoustic surface wave velocity at $T_{\mathrm{c}}$ and significant hardening at $T^{*}<T_{\mathrm{co}}$. The observed softening is ascribed to the possible softening of the elastic constant $\mathrm{C}_{44}$ below $T_{\mathrm{c}}$. The stiffening of surface sound velocity below $T_{\text {co }}$ is attributed to the strong electron-lattice coupling due to the static Jahn-Teller lattice distortion in the COI state.

\section{Experimental details}

Polycrystalline powder of $\mathrm{Nd}_{0.5} \mathrm{Sr}_{0.5} \mathrm{MnO}_{3}$ were prepared by the solid-state reaction of stoichiometric amounts of neodymium acetate, strontium carbonate and manganese dioxide. The initial materials were grounded and heated at $1000{ }^{\circ} \mathrm{C}$ for $60 \mathrm{~h}$ with two intermediate grindings. The sample was further heated at $1200{ }^{\circ} \mathrm{C}$ for another $48 \mathrm{~h}$. The polycrystalline powder was filled in a latex tube and compressed using a hydrostatic pressure of 5 tons. The rods thus obtained were sintered at $1400{ }^{\circ} \mathrm{C}$ for $24 \mathrm{~h}$. The X-ray powder diffraction pattern showed the sample to be monophasic. These rods were then subjected to floating zone melting technique, which employs SC-M35HD double reflector infrared image furnace (Nichiden Machinery Ltd, Japan). The growth rate and rotation speeds employed in all the experiments were $10 \mathrm{~mm} / \mathrm{h}$ and $30 \mathrm{rpm}$, respectively. The system was subjected to $21 / \mathrm{min}$ of air. The resistivity and magnetic susceptibility measurement show the expected transitions as reported in the literature [11]. The Laue diffraction pattern showed streaking and was difficult to index to a particular plane of a single crystal. The appearance of texture in the Laue pattern indicates that our crystal is not a true single crystal and has a lots of twin boundaries. The crystalline ingot was cut along an arbitrary plane and was mechanically polished using diamond suspension.

Brillouin spectra were recorded in $180^{\circ}$-back-scattering geometry with an incident angle of $45^{\circ}$ with respect to the surface normal. The scattered light was analyzed using a JRS Scientific instruments $(3+3)$-pass tandem Fabry-Perot interferometer equipped with a photoavalanche diode detector. The $532 \mathrm{~nm}$ line of Nd:YAG single mode solid-state diode-pumped frequency doubled laser (Model DPSS 532-400, Coherent Inc. USA) was used as the excitation source. Temperaturedependent measurements were carried out inside a closed cycle helium cryostat (CTI Cryogenics, USA). The sample temperatures were measured within an accuracy of $\pm 1 \mathrm{~K}$. The incident laser power was kept at $\sim 25 \mathrm{~mW}$ focused to a diameter of $\sim 30 \mu \mathrm{m}$. The laser heating of the sample was $20 \mathrm{~K}$, which has been corrected in plotting the data. 


\section{Results and discussion}

Fig. 1 shows Brillouin spectrum of $\mathrm{Nd}_{0.5} \mathrm{Sr}_{0.5} \mathrm{MnO}_{3}$ at room temperature which displays three modes at 5.5, 8 and $15 \mathrm{GHz}$ recorded using the free spectral range (FSR) of $25 \mathrm{GHz}$. Fig. 2(a) shows dependence of these modes frequencies $\nu_{\mathrm{s}}$ on $q \|$, the component of the wavevector parallel to the surface. It clearly shows that $\nu_{\mathrm{s}}$ is a linear function of $q \|, 2 \pi \nu_{\mathrm{s}}=V_{\mathrm{s}} q_{\|}$and thereby these modes are associated with the surface modes. The velocities obtained from the linear fit are 1955, 3182 and $5177 \mathrm{~m} / \mathrm{sec}$ for $5.5,8$ and $15 \mathrm{GHz}$ modes, respectively. Fig. 2(b) and (c) show the sinusoidal behavior of 5.5 and $8 \mathrm{GHz}$ modes with respect to the azimuthal angle $(\phi)$ (see inset of Fig. 2(a)). The data in Fig. 2 suggest that the modes at 5.5 and $8 \mathrm{GHz}$ are associated with the surface Rayleigh wave (SRW) and pseudo-surface acoustic wave (PSAW), respectively. The surface mode frequency of $15 \mathrm{GHz}$ is roughly 3 times that of SRW and it appears only along certain orientation of the crystal. Further its linewidth is much larger as compared to the other two surface modes. These results imply that the $15 \mathrm{GHz}$ mode is associated with the high velocity pseudosurface acoustic (HVPSAW) wave [25]. In comparison to SRW whose pointing vector lies parallel to the free surface,

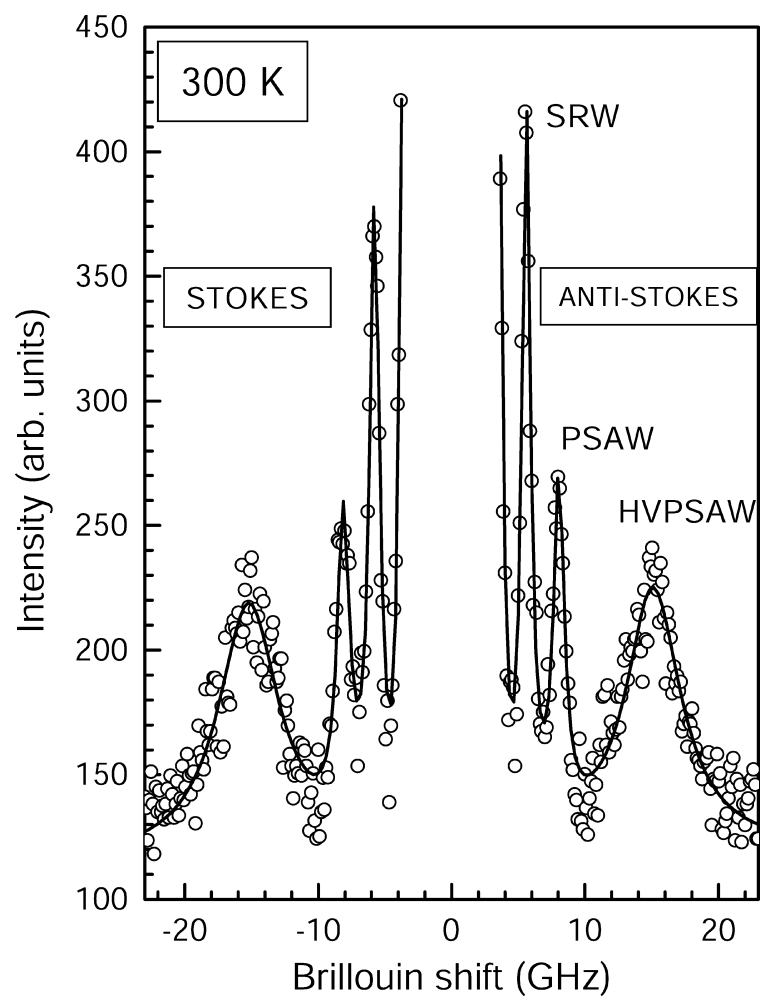

Fig. 1. Room temperature Brillouin spectrum of $\mathrm{Nd}_{0.5} \mathrm{Sr}_{0.5} \mathrm{MnO}_{3}$ recorded with the FSR $25 \mathrm{GHz}$. The solid lines are Lorentzian fit to the data with an appropriate background.
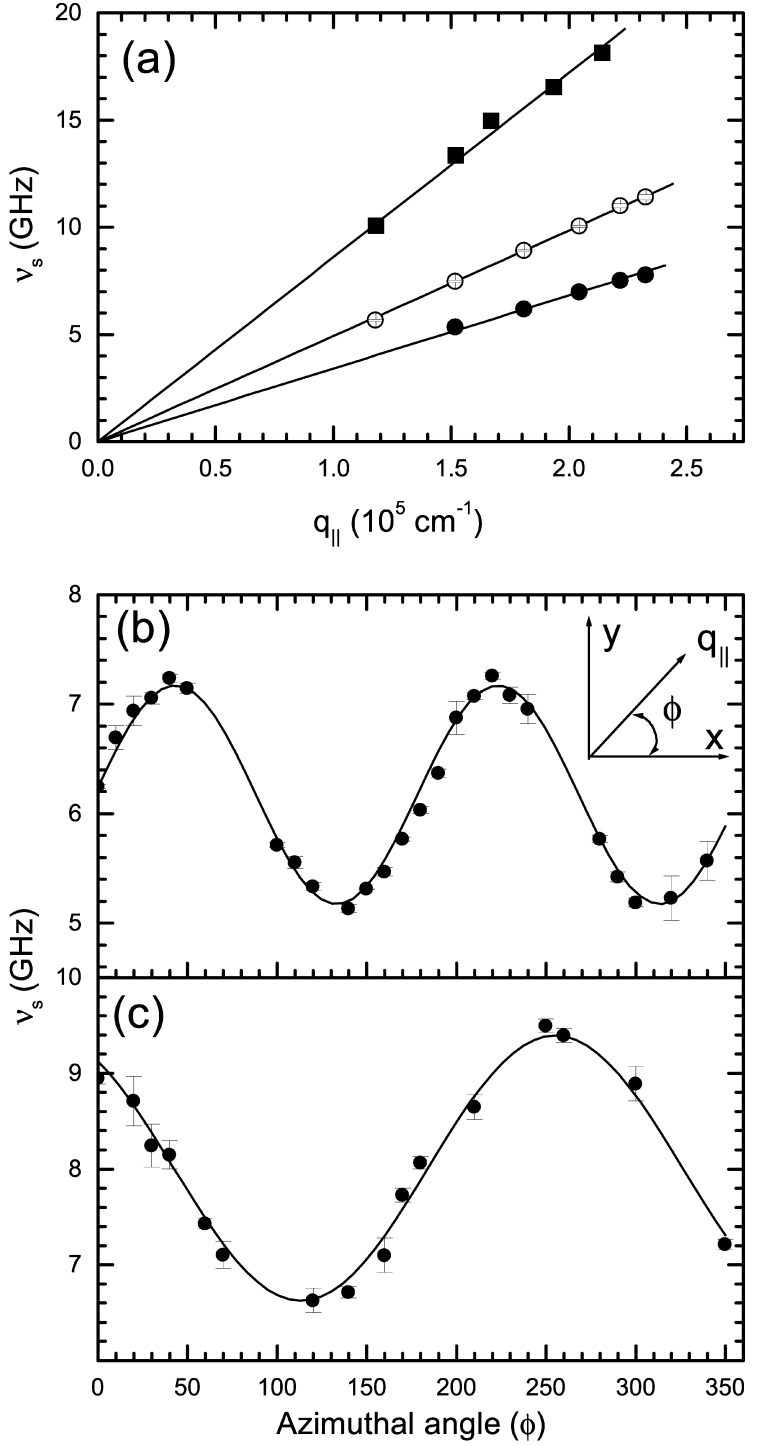

Fig. 2. (a) The frequency dependence of 5.5, 8 and $15 \mathrm{GHz}$ modes as a function of $\vec{q}_{\|}$. The solid lines are fit the data. The slope of the line is the surface velocity. (b) and (c) the azimuthal angle $(\phi)$ dependence of the 5.5 and $8 \mathrm{GHz}$ modes, respectively. The solid lines are sin fit to the data. Inset in (b) shows the definition of $\phi$ where $\mathrm{x}$ is parallel to the scattering plane.

both PSAW and HVPSAW radiate energy into the bulk and get attenuated due to their decay into bulk phonons.

Inset of Fig. 3(a) shows the temperature dependence of the SRW in cooling (solid circles) as well as heating (open circles) runs where some hysteresis can be clearly seen. The difference in the frequencies $\Delta \nu$, defined as $\Delta \nu=\nu_{\mathrm{S}}(T)-$ $\nu_{\mathrm{s}}(300 \mathrm{~K})$ and normalized with respect to the value $\nu_{\mathrm{s}}$ at $300 \mathrm{~K}$ are plotted as a function of temperature for the cooling cycle for the SRW (Fig. 3(a)) and the PSAW (Fig. 3(b)).An interesting observation is that a new mode at 


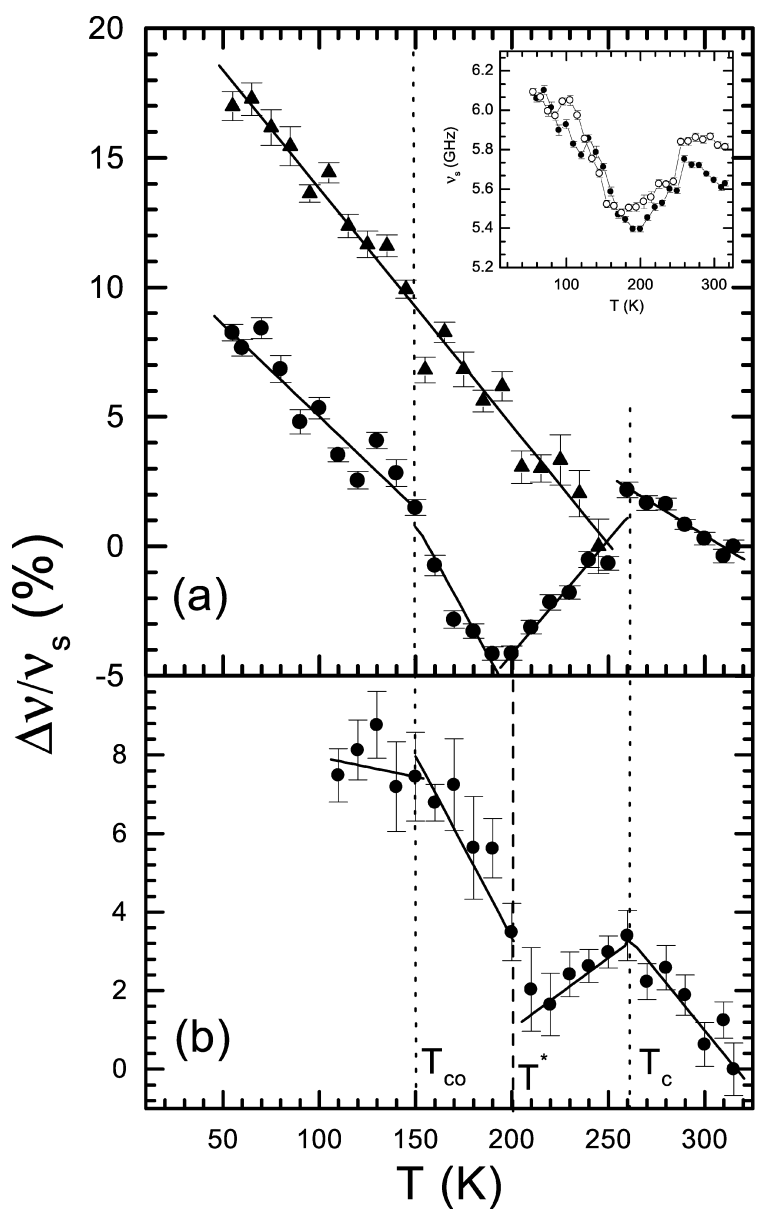

Fig. 3. Percentage change of the sound velocity as a temperature: (a) $5.5 \mathrm{GHz}$ mode (solid circles) and the new mode (up triangles) and (b) $8 \mathrm{GHz}$ mode. The solid lines are linear fit to the data. Inset in (a) shows the SRW mode frequency as a function of temperature for both cooling (solid circles) and heating (open circles) cycles.

$\sim 6.5 \mathrm{GHz}$ appears at $T_{\mathrm{c}}$. The temperature dependence of this new mode is also shown in Fig. 3(a) (filled triangles). The origin of this new mode is not clear at this point. Most interestingly, the temperature dependence of the HVPSAW mode, is very different from the other two surface modes, as seen in Fig. 4(a). The temperature dependence of the linewidth $(\Gamma)$ of the HVPSAW mode is also anomalous, namely, that $\Gamma$ increases with the decrease in temperature (Fig. 4(b)). Another curious observation is the temperature dependence of the intensity of the SRW mode, shown in Fig. 5 , which is similar to the temperature dependence of its frequency (Fig. 3(a)). We note that the $5.5 \mathrm{GHz}$ mode shows a substantial variation in intensity across both the $T_{\mathrm{c}}$ and $T_{\text {co }}$ as shown in Fig. 5. The $8 \mathrm{GHz}$ and the new mode do not show any observable change in intensity. The modes are weak compared to $5.5 \mathrm{GHz}$ mode. We will now attempt to understand the observed features shown in Figs. 3-5.

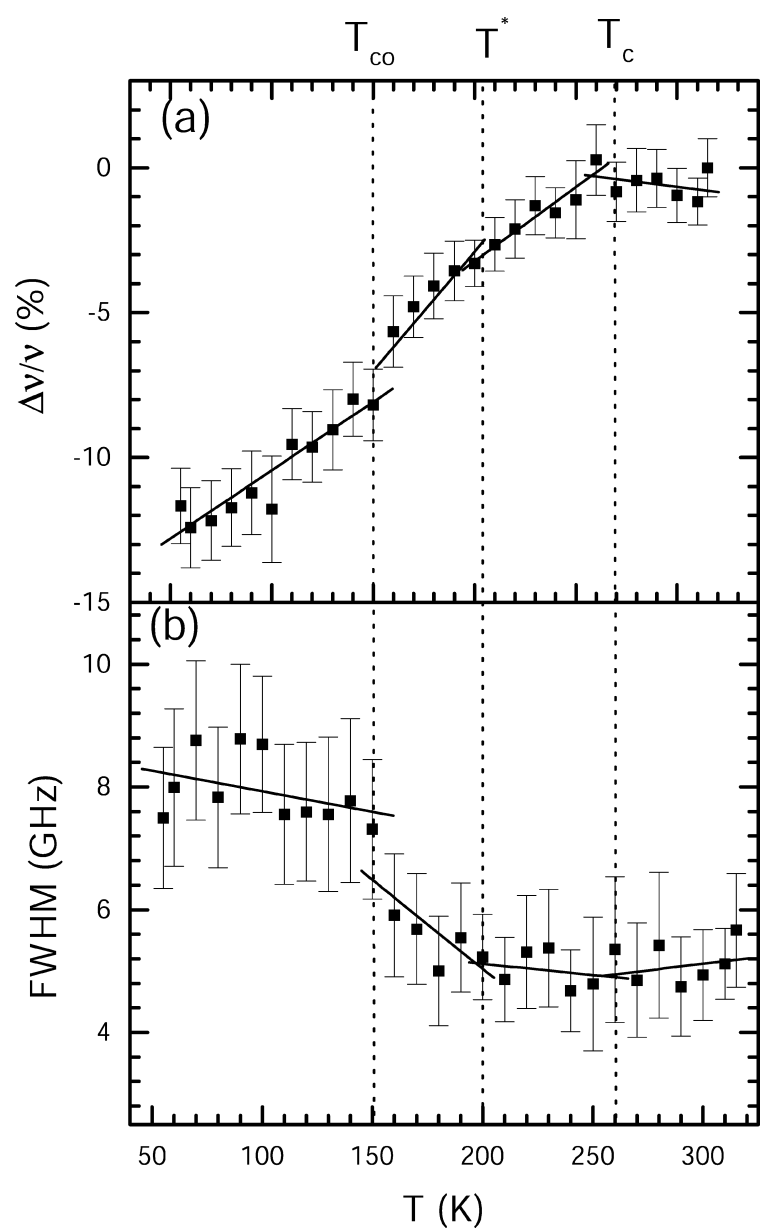

Fig. 4. (a) Percentage change of the sound velocity and (b) the fullwidth at half-maxima (FWHM) of $15 \mathrm{GHz}$ mode as a function temperature. The solid lines are linear fit to the data.

For simplicity, we assume orthorhombic $\mathrm{Nd}_{0.5} \mathrm{Sr}_{0.5}$ $\mathrm{MnO}_{3}$ to be close to a cubic system as the orthorhombic distortions over and above the cubic structure are small. Hence the sound velocity associated with the surface acoustic waves are a function of the three elastic constants, namely, $\mathrm{C}_{11}, \mathrm{C}_{12}$ and $\mathrm{C}_{44}$. A small hardening of the sound velocities for both the SRW and PSAW modes as shown in Fig. 3(a) and (b), respectively, between $300 \mathrm{~K}$ and $T_{\mathrm{c}}$ are related to the hardening of the elastic constant $\mathrm{C}_{11}$ in the PI phase with a decrease in temperature. The longitudinal sound velocity generally shows hardening below $T_{\mathrm{c}}$ in the manganites family compounds. The origin of the hardening of the sound velocity below $T_{\mathrm{c}}$ should be same in all of these materials exhibiting MI transition. It has been suggested [26] that the mode frequency increases below $T_{\mathrm{c}}$ due to the decrease in the electron-phonon coupling arising from the high mobility of carriers in the metallic state. In our study, a pronounced softening of the SRW and PSAW velocities is seen below $T_{\mathrm{c}}$ (Fig. 3(a) and (b)). To our knowledge, there is 


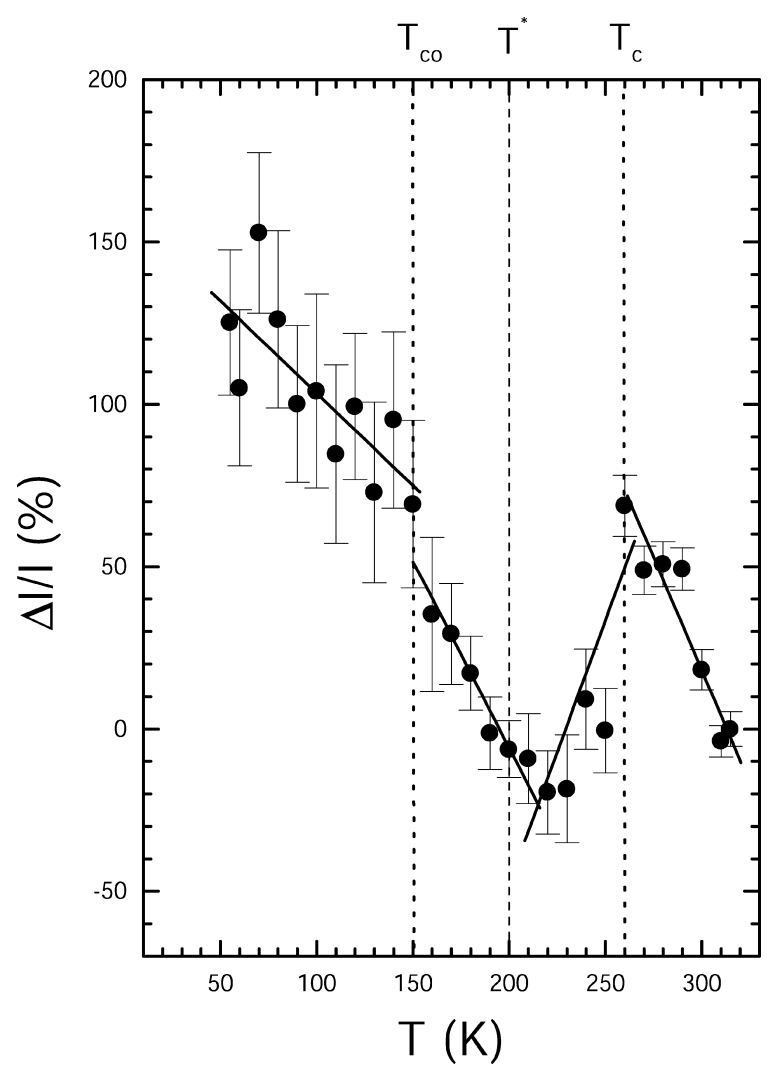

Fig. 5. The temperature dependence of the intensity of the $5.5 \mathrm{GHz}$ mode. The lines are the linear fit to the data.

no available elastic constants data on $\mathrm{Nd}_{0.5} \mathrm{Sr}_{0.5} \mathrm{MnO}_{3}$ in the literature. Therefore, we have taken the reported elastic constants data of $\mathrm{La}_{0.835} \mathrm{Sr}_{0.165} \mathrm{MnO}_{3}$ (Fig. 6(a)), which also shows a MI transition at $260 \mathrm{~K}$ [27]. Based on this data we try to understand how $\nu_{\mathrm{S}}(\mathrm{SRW})$ can soften below $T_{\mathrm{c}}$. Using Green's function method [28] we have generated the surface mode spectrum with the values of the elastic constants $\mathrm{C}_{11}$, $\mathrm{C}_{12}$ and $\mathrm{C}_{44}$ (all solid lines) of $\mathrm{La}_{0.835} \mathrm{Sr}_{0.165} \mathrm{MnO}_{3}$. The calculated SRW velocity shows the temperature dependence as shown by the solid line in Fig. 6(b). While computing the SRW frequency using Green's function method, we have seen that the frequency is more sensitive to changes in $\mathrm{C}_{44}$ than in $\mathrm{C}_{12}$. In order to get softening of the SRW frequency, we assume a softening of $\mathrm{C}_{44}$, shown by the dotted line in Fig. 6(a) and marked as $\mathrm{C}_{44}^{*}$, along with the earlier temperature dependence of $\mathrm{C}_{11}$ and $\mathrm{C}_{12}$. This possibility does result in the softening of $\nu_{\mathrm{s}}$ below $T_{\mathrm{c}}$, as shown by the dotted line in Fig. 6(b). To get an exact matching of the calculated frequencies with the experimental values, one need to know the actual temperature dependence of all the elastic constants for $\mathrm{Nd}_{0.5} \mathrm{Sr}_{0.5} \mathrm{MnO}_{3}$.

The SRW sound velocity shows a minima at $\sim 200 \mathrm{~K}$, which is nearly at the center of $T_{\mathrm{c}}$ and $T_{\mathrm{co}}$. We denote this temperature as $T^{*}$ (Fig. 3), where the SRW and PSAW

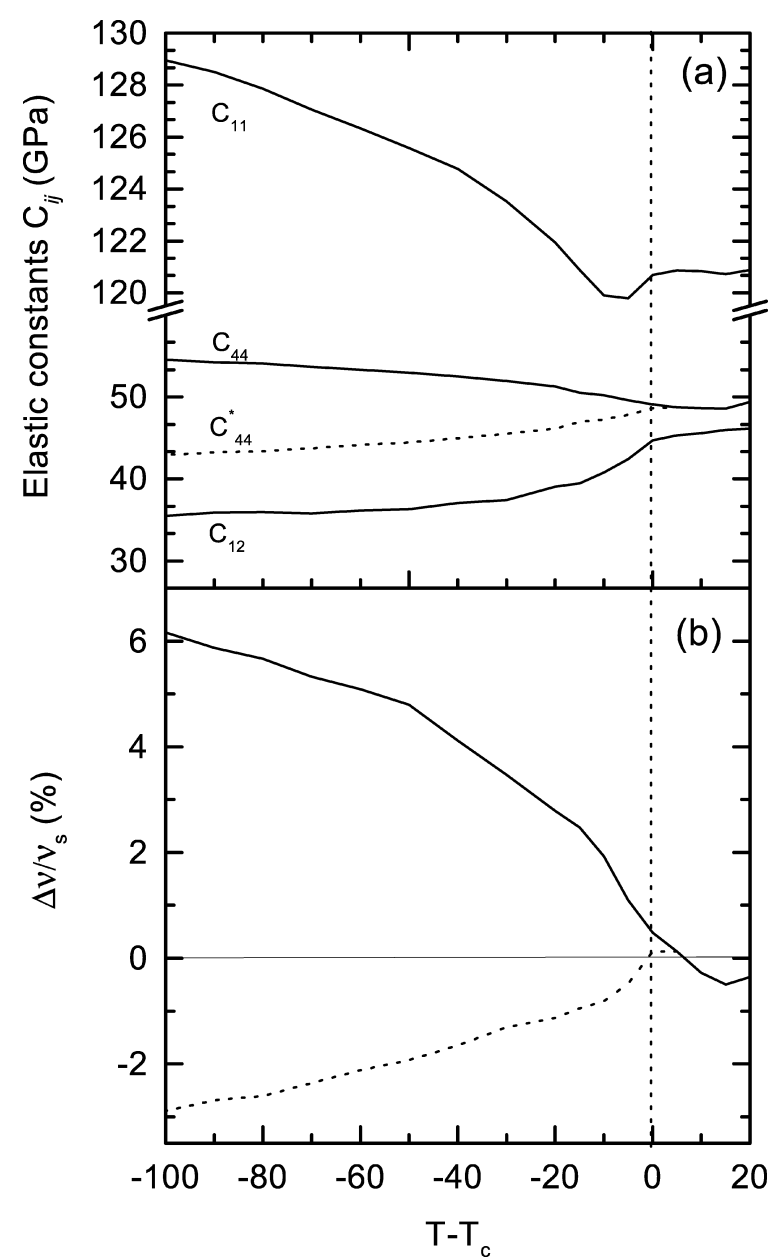

Fig. 6. (a) Elastic constants of $\mathrm{La}_{0.835} \mathrm{Sr}_{0.165} \mathrm{MnO}_{3}$ Ref. [27] plotted as a function of $T-T_{\mathrm{c}}$ (solid lines). $\mathrm{C}_{44}^{*}$ is the guess value of $\mathrm{C}_{44}$ below $T_{\mathrm{c}}$ (dotted line). (b) Percentage change of the sound velocity calculated from the above elastic constants data (solid line) and replacing $\mathrm{C}_{44}$ by $\mathrm{C}_{44}^{*}$ (dotted line).

velocities again take an upturn. We believe that this can be related to the appearance of the A-type anti-ferromagnetic phase at $\sim 200 \mathrm{~K}$ in $\mathrm{Nd}_{0.5} \mathrm{Sr}_{0.5} \mathrm{MnO}_{3}$ [19] or to the coexistence of the charge ordered phase along with the FMM phase. Lee and Min [26] have explained the hardening of the bulk longitudinal acoustic phonon velocity (related to $\mathrm{C}_{11}$ ) by coupling of the acoustic phonon with the dynamics of charge ordering. In their model, the change ordering was modeled by repulsively interacting small polarons. We suggest that the increase of SRW velocity below $T_{\mathrm{c}}$ is related to the same mechanism of Lee and Min [26].

Temperature behavior of the frequency and linewidth of the $15 \mathrm{GHz}$ mode associated with HVPSAW is rather anomalous. The frequency continuously decreases with definite slope changes across $T_{\mathrm{c}}, T^{*}$ and $T_{\text {co }}$ as shown in Fig. 4(a), where the solid lines are piece-wise linear fits to the data. This unusual softening of this HVPSAW mode and the 
anomalous increase in linewidth (Fig. 4(b)) are not understood at present. We suggest that it may be related to the opening up of new decay channels for the HVPSAW mode by spin-phonon coupling.

In summary, we have carried out Brillouin scattering measurements of the sound velocities of the surface modes of $\mathrm{Nd}_{0.5} \mathrm{Sr}_{0.5} \mathrm{MnO}_{3}$ across $T_{\mathrm{c}}$ and $T_{\text {co. }}$. The unusual softening of the SRW and PSAW sound velocities immediate below $T_{\mathrm{c}}$ is associated with the softening of the $\mathrm{C}_{44}$ elastic constant. An increase of the surface mode velocity at $T^{*}$ can be related to the appearance of A-type anti-ferromagnetic phase or the existence of the charge ordered phase along with the FMM phase. The significant hardening of the sound velocity below $T^{*}$ and $T_{\text {co }}$ imply a strong electron-lattice coupling via Jahn-Teller distortions. It will be interesting to measure the temperature-dependence of $\mathrm{C}_{44}$ which is expected to show softening below $T_{\mathrm{c}}$. A quantitative understanding of the anomalous temperature-dependence of frequency and linewidth of the HVPSAW mode is yet to be developed.

\section{Acknowledgements}

We thank Prof. C.N.R. Rao for invaluable continued support and encouragement. A.K.S. thanks Department of Science and Technology (DST) for assistance. M.M.S. would like to thank Council of Scientific and Industrial Research (CSIR), India for the fellowship.

\section{References}

[1] S. Jin, T.H. Tiefel, M. McCormack, R.A. Fastnacht, R. Ramesh, L.H. Chen, Science 264 (1994) 413.

[2] C. Zener, Phys. Rev. 82 (1951) 403.

[3] P.W. Anderson, H. Hasegawa, Phys. Rev. 100 (1955) 675.

[4] P.G. de Gennes, Phys. Rev. 100 (1955) 564.

[5] A.J. Millis, P.B. Littlewood, B.I. Shraiman, Phys. Rev. Lett. 74 (1995) 5144.

[6] A.J. Millis, B.I. Shraiman, R. Mueller, Phys. Rev. Lett. 77 (1996) 175.

[7] A.P. Ramirez, P. Schiffer, S.-W. Cheong, C.H. Chen, W. Bao,
T.T.M. Palstra, P.L. Gammel, D.J. Bishop, B. Zegarski, Phys. Rev. Lett. 76 (1996) 3188.

[8] J.B. Goodenough, J.S. Zhou, Nature (London) 386 (1997) 229.

[9] S.J.L. Billinge, R.G. DiFrancesco, G.H. Kwei, J.J. Neumeier, J.D. Thompson, Phys. Rev. Lett. 77 (1996) 715.

[10] G. Zhao, K. Conder, H. Keller, K.A. Muller, Nature (London) 381 (1996) 676.

[11] H. Kuwahara, Y. Tomioka, A. Asamitsu, Y. Moritomo, Y. Tokura, Science 270 (1995) 961.

[12] E.J.W. Verwey, P.W. Haayman, Physica VIII (9) (1941) 979.

[13] R. Kajimoto, H. Yoshizawa, H. Kawano, H. Kuwahara, Y. Tokura, K. Ohoyama, M. Ohashi, Phys. Rev. B 60 (1999) 9506.

[14] Y. Tokura, H. Kuwahara, Y. Moritomo, Y. Tomioka, A. Asamitsu, Phys. Rev. Lett. 76 (1996) 3184.

[15] A. Biswas, A. Arulraj, A.K. Raychaudhuri, C.N.R. Rao, J. Phys.: Condens. Matter 12 (2000) L101.

[16] S. Mori, C.H. Chen, S.-W. Cheong, Nature 392 (1998) 473.

[17] M. Uehara, S. Mori, C.H. Chen, S.-W. Cheong, Nature (London) 399 (1999) 560.

[18] S.-W. Cheong, P.A. Sharma, N. Hur, et al., Physica 318B (2002) 39

[19] C. Ritter, R. Mahendiran, M.R. Ibarra, L. Morellon, A. Maignan, B. Raveau, C.N.R. Rao, Phys. Rev. B 61 (2000) R9229.

[20] J. Geck, D. Bruns, C. Hess, R. Klingeler, P. Reutler, M.V. Zimmermann, S.-W. Cheong, B. Bchner, Phys. Rev. B 66 (2002) 184407.

[21] V. Kiryukhin, B.G. Kim, T. Katsufuji, J.P. Hill, S.-W. Cheong, Phys. Rev. B 63 (2001) 144406.

[22] T. Asaka, Y. Anan, T. Nagai, S. Tsutsumi, H. Kuwahara, K. Kimoto, Y. Tokura, Y. Matsui, Phys. Rev. Lett. 89 (2002) 207203.

[23] M. Martin, G. Shirane, Y. Endoh, K. Hirota, Y. Moritomo, Y. Tokura, Phys. Rev. B 54 (2001) 14285.

[24] S. Zvyagin, H. Schwenk, B. Lthi, K.V. Kamenev, G. Balakrishnan, D.M. Paul, V.I. Kamenev, G.Y. Pashkevich, Phys. Rev. B 62 (2002) R6104.

[25] G. Carlotti, D. Fioretto, L. Giovannini, F. Nizzoli, G. Socino, L. Verdini, J. Phys.: Condens. Matter 4 (1992) 257.

[26] J.D. Lee, B.I. Min, Phys. Rev. B 55 (1997) 12454. J.D. Lee, B.I. Min, Phys. Rev. B 55 (1997) 14713.

[27] H. Hazama, Y. Nemoto, T. Goto, A. Asamitsu, Y. Tokura, Physica B 281/282 (2000) 487.

[28] X. Zhang, J.D. Cummins, A.G. Every, P.R. Stoddart, W. Pang, T.E. Derry, Phys. Rev. B 58 (1998) 13677. 